\title{
Antibiotic Resistance of Vibrio parahaemolyticus Isolated from Cockles and Shrimp Sea Food Marketed in Selangor, Malaysia
}

\section{Saleh MY Al-Othrubi ${ }^{\star 1}$, Cheah Yoke Kqueen ${ }^{2}$, Hamed Mirhosseini ${ }^{1}$, Yousr Abdul Hadi ${ }^{3}$ and Son Radu}

${ }^{1}$ Center of Excellence for Food Safety Research, Faculty of Food Science and Technology, University Putra Malaysia, Malaysia

${ }^{2}$ Department of Biomedical Sciences, Faculty of Medicine and Health Sciences, University Putra Malaysia, Malaysia

${ }^{3}$ Department of Cell and Molecular Biology, Faculty of Biotechnology, University Putra Malaysia, 43400 UPM, Serdang, Malaysia

\begin{abstract}
Introduction: The main aim of this study is to determine the antibiotic profile of $V$. parahaemolyticus gastroenteritis associated with the consumption of contaminated shrimp and cockles marketed in Selangor Malaysia $V$. parahaemolyticus is the leading cause of seafood-associated gastroenteritis in Asian Countries typically is associated with the consumption of raw shellfish and oysters specially shrimp and cockles. Rapid, sensitive and specific detection methods are needed to control $V$. parahaemolyticus infections. We describe a recognized the pathogenic $V$. parahaemolyticus in shrimp and cockles that will be the risk of gastroenteritis associated with the consumption of seafood marketed in Malaysia.

Methods: This study was carried out between July 2011 and August 2013 at the Center of Excellence for Food Safety Research, Faculty of Food Science and Technology, Faculty of Medicine and Health Sciences, Department of Biomedical Sciences, and Faculty of Biotechnology, Dep. of Cell and Molecular Biology, University Putra Malaysia and other centers as collaboration. The seafood samples were collected from different markets and more than 400 samples from shrimp and cockles were investigated for detection and isolation of $V$. parahaemolyticus. CHROMagar Vibrio and TCBS agar media were used for fast detection and isolation of $V$. parahaemolyticus isolates. PCR based methods targeted to tox $R$ regulatory gene, $t / h$ the species and family gene, $t d h$ and th the virulence genes were extensively used. The antibiotic susceptibility testing of $65 \mathrm{~V}$. parahaemolyticus isolates recovered from retail shrimp and cockles seafood were determined with four types of E-test antibiotic strips.
\end{abstract}

Results: All the 65 isolates were positive to tox $R$ and $t / h$ genes. Out of 65 isolates, only eight isolates $(12.31 \%)$ were positive for $t d h$ virulence gene isolated form cockles and shrimp ( 3 isolates from shrimp and 5 isolates from cockles), whereas twenty six (40\%) isolates were positive for trh virulence gene isolated from shrimp and cockles (9 from shrimp and 17 from cockles). This result indicates high occurrence of $t d h+$ and $t r h+$ isolates in shrimp and cockles marketed in Malaysia. None of the isolates tested possess both virulence genes. For the antibiotic E-test susceptibility test, overall, V. parahaemolyticus is remained susceptible to tetracycline (97\%). A slight increase in the susceptibility of tetracycline is observed from 2011 to 2013 . While reduced susceptibility was detected only in $V$. parahaemolyticus for ampicillin. The mean of MIC of the isolates toward ampicillin is increased from $64 \mu \mathrm{g} / \mathrm{ml}$ in 2011 to $128 \mu \mathrm{g} / \mathrm{ml}$ in year 2013. The current study demonstrates a high risk of pathogenic $V$. parahaemolyticus in the shrimp and cockles marketed in Selangor Malaysia.

Conclusions: The potential risk of $V$. parahaemolyticus infection due to the consumption of contaminated seafood in Malaysia should not be neglected. The increased resistance of ampicilin from our studies in Malaysia since 2004 to 2013 could be in indication of antibiotic abuse in clinical and agricultural used of ampicilin in Malaysia.

Keywords: Vibrio parahaemolyticus; Antibiotic E-test; toxR; tlh; tdh; trh; CHRO magar vibrio

\section{Introduction}

V. parahaemolyticus, a gram-negative marine bacterium, is a major food-borne pathogen that causes acute human gastroenteritis associated with the consumption of seafood. V.parahaemolyticus is a gram-negative halophilic bacterium and is responsible for human gastroenteritis worldwide. Sporadic cases and outbreaks of V. parahaemolyticus occur regularly in Asia and as well as in other countries [1-5]. Cases of $V$. parahaemolyticus were mostly sporadic and associated with diverse serovars. However, the emergence of a pandemic serovar O3:K6 in 1996 has changed the epidemiology abruptly and has since been accounted for many cases of V. parahaemolyticus outbreak worldwide [6-8].

Not all strains of V. parahaemolyticus cause illness in humans; in fact, the majority of strains isolated from the environment or seafood are not pathogenic. The pathogenic strains of $V$. parahaemolyticus are those that produce Thermostable Direct Haemolysin (TDH) toxin $[9,10] . T D H$ is an enzyme that lyses human red blood cells on Wagatsuma blood agar plates, which is referred to as the Kanagawa phenomenon positive $(\mathrm{KP}+)$. KP is a type of beta-hemolysis induced by $T D H$ toxin encoded by the $t d h$ gene. The role of the toxin in illness is not known and $90 \%$ of the $t d h$ positive strains isolated from clinical cases show hemolysis, while only 1 to $2 \%$ of the strains of environmental origin are KP positive [11]. Another toxin produced by KP-negative $V$. parahaemolyticus strains [12] is the TDH-Related hemolysin (TRH) toxin encoded by trh gene. These isolates which are urease positive

*Corresponding author: Saleh MY Al-Othrubi, Center of Excellence for Food Safety Research, Faculty of Food Science and Technology, University Putra Malaysia, 43400 UPM, Serdang, Malaysia, Tel: +60122201392; E-mail: medmicsm2005@gmail.com

Received April 02, 2014; Accepted April 28, 2014; Published May 10, 2014

Citation: Al-Othrubi SMY, Kqueen CY, HMirhosseini CY, Hadi YA, Radu S (2014) Antibiotic Resistance of Vibrio parahaemolyticus Isolated from Cockles and Shrimp Sea Food Marketed in Selangor, Malaysia. Clin Microbial 3: 148. doi:10.4172/23275073.1000148

Copyright: @ 2014 Al-Othrubi SMY, et al. This is an open-access article distributed under the terms of the Creative Commons Attribution License, which permits unrestricted use, distribution, and reproduction in any medium, provided the original author and source are credited. 
can cause skin infection when the injured skin is exposed into sea water leading to wound infections and septicemia $[11,13,14]$. To date pathogenic strains containing $t d h$ and/or $t r h$ genes have been detected with low frequency (usually 0.3 to $3 \%$ ) in the total $V$. parahaemolyticus environmental population $[15,16]$

Vibrio is generally considered to be highly susceptible to most clinically used antimicrobials [17]. However, during the past few decades, antimicrobial resistance has emerged and evolved in many bacterial genera due to the excessive use of antimicrobials in human, agriculture, and aquaculture systems $[18,19]$. This emerging issue has gain great concern due to increase resistance of pathogenic $V$. parahaemolyticus towards clinically used antimicrobials. Tetracycline [20] and an alternative treatments of combinations of expandedspectrum cephalosporins (e.g., ceftazidime) and doxycycline or a fluoroquinolone alone [21] have been recommended as the antimicrobial of choice for treatment of severe Vibrio infections.

In Malaysia, $V$. parahaemolyticus is naturally occurring in the marine coastal region of Malaysia. It is prevalent in the tropical marine environment in all seasons and can cause seafood-borne gastroenteritis. $V$. parahaemolyticus has been recognized as one of the causative agents in the frequent institutional food poisoning incidences in Malaysia [22]. The occurrence of $V$. parahaemolyticus in seafood is getting intense attention in Malaysia due to frequent rejection of seafood export to EU countries [22]. Due to this, the first national risk assessment of $V$. parahaemolyticus in seafood was initiated and carried out to control and manage this seafood-borne pathogen in Malaysia [22,23]. Surveillance study carried out in Malaysia showed high prevalent contamination of pathogenic Vibrio spp. in retail seafoods in the country throughout the year and suggest that there is a need for adequate consumer protection measures [24].

The $V$. parahaemolyticus food poisoning incidence in Malaysia is considerably high. However, the occurrence of pathogenic strains in shrimp and cockles, and antibiotic resistance of $V$. parahaemolyticus strains is not well documented and studied. This study aims to provide an insight into the prevalence of $V$. parahaemolyticus strains (TDH and/or TRH) in the marine environment and retail seafood and the antibiotic resistance profile of $V$. parahaemolyticus isolated from 2004 and recently for this study from 2011 to 2013.

The presence of thermostable direct hemolysin $T D H$ which coded by $t d h$ gene is a proven virulence factor [25] [26], and TDH occurs in over $90 \%$ of clinical strains of $V$. parahaemolyticus $[4,27,28]$. Most literatures reported a low prevalence of (less than 1\%) $t d h$ gene in the isolates from environmental and seafood samples [29-31], except in a limited study in Grays Harbor, Washington [32]. A proposed virulence factor, the $T D H$-related hemolysin (TRH), encoded by the gene trh has been discovered also in clinical stains of $V$. parahaemolyticus lacking $t d h[12,33]$. Most clinical isolates from the U.S. Pacific Coastal have been reported to possess both $t d h$ and trh [13]. Our findings are in agreement with Wong [4] in which some environmental isolates were found to possess trh genes only but very low $t d h$ positive isolates. None of the isolates collected in this study possess both $t d h$ and $t r h$ genes suggests $t d h$ gene is mainly contained within clinical strains of $V$. parahaemolyticus. In the previous studies, the prevalence of pathogenic $V$. parahaemolyticus in the marine environment and retail seafood is relatively low. Nonetheless, there is still a potential risk of $V$. parahaemolyticus outbreak or infection through consumption of the contaminated seafood.

For the antibiotics, treatment of severe Vibrio infections,
Tetracycline has been recommended as the antimicrobial of choice $[20,34]$, and alternative treatments are combinations of expandedspectrum cephalosporins (e.g., ceftazidime) and doxycycline or a fluoroquinolone alone [21,34]. Trimethoprim-sulfamethoxazole plus an aminoglycoside is used to treat children in whom doxycycline and fluoroquinolones are contraindicated [14,35]. Traditionally, Vibrio is considered highly susceptible to virtually all antimicrobials [17]. During the past few decades, however, antimicrobial resistance has emerged and evolved in many bacterial genera due to the excessive use of antimicrobials in human, agriculture, and aquaculture systems $[18,19,34]$.

E-test and Minimum Inhibitory Concentrations (MIC) of antibiotics are routinely determined by broth or agar serial dilution methods or by agar diffusion methods. More recently, e-test was developed to reduce the time, labor, and materials used in MIC determination assays. E-test is based on arraying a concentration gradient of each antibiotic on a polymer strip. Concentration values are marked on the other side of the strip so that one can easily locate corresponding concentrations. E-strips, also known as "epsilometers", are commercially prepared by micro dispersing robotic machines that can deliver nanoliter volumes of antibiotic concentration along the strip. Each antibiotic strip is laid on the surface of an inoculated agar plate. An elliptical zone of inhibition develops with the broad end at the top of the strip with the highest antibiotic concentration and the narrowest end at the lowest amount of antibiotic that can inhibit bacterial growth, i.e., minimum inhibitory concentration. Several different antibiotic e-strips can be tested simultaneously on the same agar plate. Therefore MIC's can be determined for many antibiotics in a single step with no need for dilution in broth or agar. Also, e-test is applied routinely as a "culture sensitivity test" in some medical laboratories in place of the traditional Kirby-Bauer method. In addition to reduction of time and effort, e-test yields sensitivity test results in quantitative terms which make interpretation of results more precise and easier than routine methods [36].

\section{Methodology}

We used the rapid methods to detect and isolate V.parahaemolyticus from the expected and selected contaminated seafood and any environmental or (clinical) samples by using CHROMagar Vibrio medium, the highest selective medium for Vibrio, and PCR based method targeted to VP-toxR species-specific regulatory gene and $t d h /$ trh the virulence genes to detect the pathogenic $V$. parahaemolyticus isolates.

The seafood samples were collected from different markets and more than 400 samples from shrimp and cockles seafood were investigated for detection and isolation of V. parahaemolyticus. CHROMagar Vibrio and TCBS agar media were used for fast detection and isolation of $V$. parahaemolyticus isolates. A total of $65 \mathrm{~V}$. parahaemolyticus isolates were obtained from shrimp and cockles ( 27 isolates from shrimp and 38 isolates from cockles). Three reference strains were used in this study as positive and negative controls, namely VP2053 and PV1808 $\left(t d h^{+}\right)$ $\left.t r h^{-}\right)$and VP1896 $\left(t d h^{-} / t r h^{+}\right)$as positive control and Escherichia coli ATCC25922 as non-vibrios (negative control). All V. parahaemolyticus strains were maintained on cryogenic beads at $-80^{\circ} \mathrm{C}$. The working cultures of $V$. parahaemolyticus were maintained on Luria Bertani (LB) broth culture with $15 \%$ Glycerol at $-30^{\circ} \mathrm{C}$ for no longer than 3 months or at $-80^{\circ} \mathrm{C}$ for longer.

All the isolates were grown overnight on Luria-Bertani (LB) agar plates supplemented with $3 \% \mathrm{NaCl}$ for $t d h$ and $t r h$ genes detection. Three 
to five colonies were scraped from the agar plates and re-suspended in $400 \mu \mathrm{l}$ of filtered sterile Milli-Q-distilled water, and boiled for $10 \mathrm{~min}$ to liberate the nucleic acid as described elsewhere [11]. The tubes were then incubated on ice for 20 min followed by centrifugation at $9000 \mathrm{x}$ g. The supernatant that contained the DNA template was transferred into new labeled sterile tubes and stored at $-30^{\circ} \mathrm{C}$ until used for PCR amplification. The $t d h$ and $t r h$ genes were amplified with the following primer sets: 5'-GGTA CTAA ATGG CTGA CATC-3' (forward) and 5'-CCAC TACC ACTC TCAT ATGC-3' (reverse) [21]; and 5'-GGCT CAAA ATGG TTAA GCG-3' (forward) and 5'-CATT TCCG CTCT CATA TGC-3' (reverse) [23], respectively. The reaction mixtures (final volume, $25 \mu \mathrm{l}$ ) contained $\mu \mathrm{l}$ of DNA template ( $50 \mathrm{ng} / \mu \mathrm{l}$ con.), $2.5 \mu \mathrm{l}$ of 10x reaction buffer ( $1^{\text {st }}$ BASE Laboratories), $4 \mu \mathrm{l}$ of $50 \mathrm{mM} \mathrm{MgCl} 2,0.25$ $\mu \mathrm{l}$ of Taq polymerase $(5 \mathrm{U} / \mu \mathrm{l}), 0.5 \mu \mathrm{l}$ of deoxynucleoside triphosphates ( $10 \mathrm{mmol}), 0.5 \mu \mathrm{l}$ of each primer $(10 \mu \mathrm{M} / \mu \mathrm{l})$, and $15.75 \mu \mathrm{l}$ of distilled water. The reactions were performed with a Gene Amp PCR system 2700 thermocycler (Bio-Rad) as follows: $4 \mathrm{~min}$ of initial denaturation at $94^{\circ} \mathrm{C}$, followed by 30 cycles of denaturation at $94^{\circ} \mathrm{C}$ for 30 s, alignment at $58^{\circ} \mathrm{C}$ for $30 \mathrm{~s}$, and extension at $72^{\circ} \mathrm{C}$ for $30 \mathrm{~s}$ and a final extension at $72^{\circ} \mathrm{C}$ for $7 \mathrm{~min}$. Positive and negative DNA controls were included in all assays. Amplified products were separated by electrophoresis in ethidium bromidestained $1.5 \%$ agarose gels in Tris-borate-EDTA (0.5x TBE) buffer at $90 \mathrm{~V}$ for $40 \mathrm{~min}$. A 100- to 1500-bp ladder (Sigma) was used as a molecular mass marker. The gels were visualized for $251 \mathrm{bp}$ ( $t d h$ gene amplicon) and 250bp (trh gene amplicon) with a UV transilluminator system and software (Bio-Rad).

All the strains were randomly selected for antibiotic susceptibility testing by using E-test strips. Five reference strains namely, VP2053, VP1896, VP1808, V. alginolyticus 2341 and Escherichia coli ATCC25922 were included in the analysis. Susceptibility testing was performed using the E-test gradient technology recommended by the National Committee for Clinical Laboratory Standard Institute (CLSI). The measurements were interpreted as resistant (R), intermediate (I) and susceptible (S) to the antibiotics according to the CLSI [37]. The antibiotic being tested in this study are Tetracycline (Tc) (MIC $0.5-2 \mu \mathrm{g} / \mathrm{ml}$ ), Cefalexin (Cx) (MIC 4-16 $\mu \mathrm{g} / \mathrm{ml}$ ), Ciprofloxacin (Ci), (MIC 0.0125-0.5 $\mu \mathrm{g} / \mathrm{ml}$ ), and Ampicillin (Am) (MIC 2-8 $\mu \mathrm{g} / \mathrm{ml}$ ) (AB BIODISK). Using a sterile cotton swabs, 3 to 5 pure colonies were picked up from fresh LB agar plate overnight cultures and inserted into a tube containing $3 \mathrm{ml}$ sterile normal saline $(0.85 \%)$ and the turbidity is adjusted to $0.5 \mathrm{McF}$ arland turbidity level. The suspension was then surface inoculated onto Mueller Hinton agar plates. The inoculated plates were allowed to air dry in laminar airflow for $10 \mathrm{~min}$ before the E-test antibiotic strips were placed on the surface carefully with sterile forceps. The plates were incubated at $37^{\circ} \mathrm{C}$ for $18-24 \mathrm{~h}$. The MIC was read at the point where the zone of growth inhibition intersected the strip.

\section{Statistical Design}

Statistical design and data analysis; A Completely Randomized Design (CRD) was considered to create different experimental treatments. In the current study, the effect of four different types of antibiotics (i.e. Tetracycline, Ampicilin, Cefalexin and Ciprofloxacin) as independent variables on total count of different strains of $V$. parahaemolyticus isolated from retail shrimp and cockle seafood was investigated. In the present study, total count of different strains of $V$. parahaemolyticus isolated from retail shrimp and cockles as percentage of sensitive and resistance $V$. parahaemolyticus were considered as response variable. In this study, cluster random sampling was employed to collect more than 400 samples from shrimp and cockles from different market in Malaysia between July 2011 and August 2013.

\section{Results}

All the 65 isolates were positive to toxR environmental regulatory gene and th family gene. Out of 65 isolates, only eight isolates (12.31\%) were positive for $t d h$ virulence gene isolated form cockles and shrimp (3 isolates from shrimp and 5 isolates from cockles), whereas twenty six (40\%) isolates were positive for trh virulence gene isolated from shrimp and cockles ( 9 from shrimp and 17 from cockles). This result indicates high occurrence of $t d h+$ and trh+ isolates in shrimp and cockles marketed in Selangor, Malaysia. None of the isolates tested possess both virulence genes.

For the E-test antibiotic susceptible testing of the selected 65 isolates of $V$. parahaemolyticus isolated from cockles and shrimp in this study revealed a high resistant in Ampicillin (63.1\%) and Cefalexin (35.4\%). In general, the isolates showed the highest susceptibility to Tetracycline (97\%) followed by Ciprofloxacin (49.3\%), (Table 1 and Figure 1). The isolates originated from retail cockles purchased over 2011 to 2013 showed the highest resistance level toward Ampicillin, Cefalexin and Ciprofloxacin compared to isolates collected from shrimp (Figure 1).

\begin{tabular}{|l|c|c|c|c|c|c|c|c|}
\hline & \multicolumn{2}{|c|}{ Tetracycline } & \multicolumn{2}{c|}{ Ampicillin } & \multicolumn{2}{c|}{ Cefalexin } & \multicolumn{2}{c|}{ Ciprofloxacin } \\
\cline { 2 - 10 } & $\%$ & \# isolates & $\%$ & \# isolates & $\%$ & \# isolates & $\%$ & \# isolates \\
\hline Sensitive & 97 & 63 & 20 & 13 & 23.1 & 15 & 49.3 & 32 \\
\hline Intermediate & 3.0 & 2 & 16.9 & 11 & 41.5 & 27 & 29.2 & 19 \\
\hline Resistance & 0.0 & 0 & 63.1 & 41 & 35.4 & 23 & 21.5 & 14 \\
\hline TOTAL & & $\mathbf{6 5}$ & & $\mathbf{6 5}$ & & $\mathbf{6 5}$ & & $\mathbf{6 5}$ \\
\hline
\end{tabular}

Table 1: Antibiotic susceptibility E-test assays for sixty five strains of $V$ parahaemolyticus isolated from shrimp and cockles marketed in Selangor Malaysia.

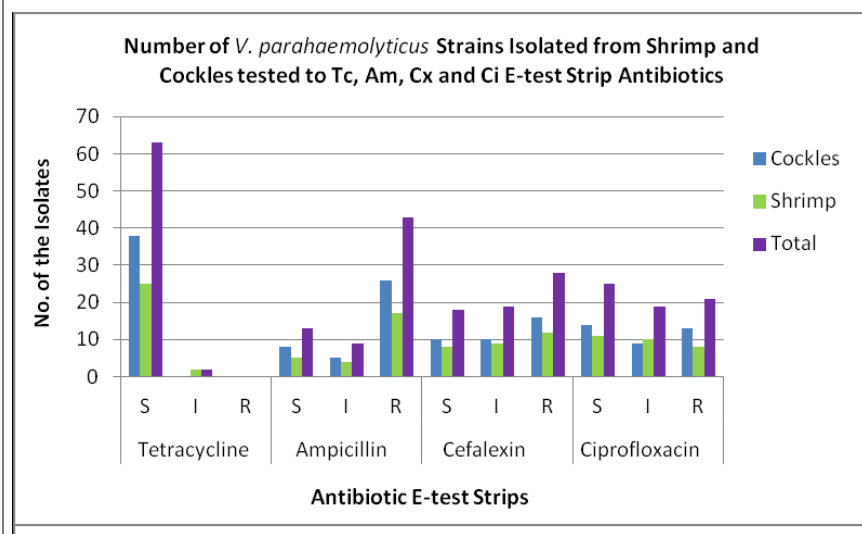

Number of Sensitive and Resistance $V$. parahaemolyticus Isolates to Tc, Am, Cx and Ci E-test strip Antibiotics

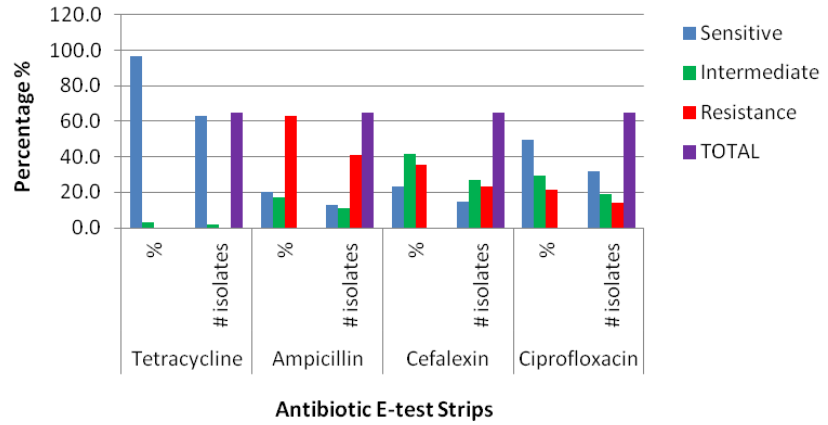

Figure 1: Tetracycline, Ampicillin, Cefalexin and Ciprofloxacin resistance of $V$. parahaemolyticus isolated from cockles and shrimp marketed in Selangor Malaysia. 
Citation: Al-Othrubi SMY, Kqueen CY, HMirhosseini CY, Hadi YA, Radu S (2014) Antibiotic Resistance of Vibrio parahaemolyticus Isolated from Cockles and Shrimp Sea Food Marketed in Selangor, Malaysia. Clin Microbial 3: 148. doi:10.4172/2327-5073.1000148
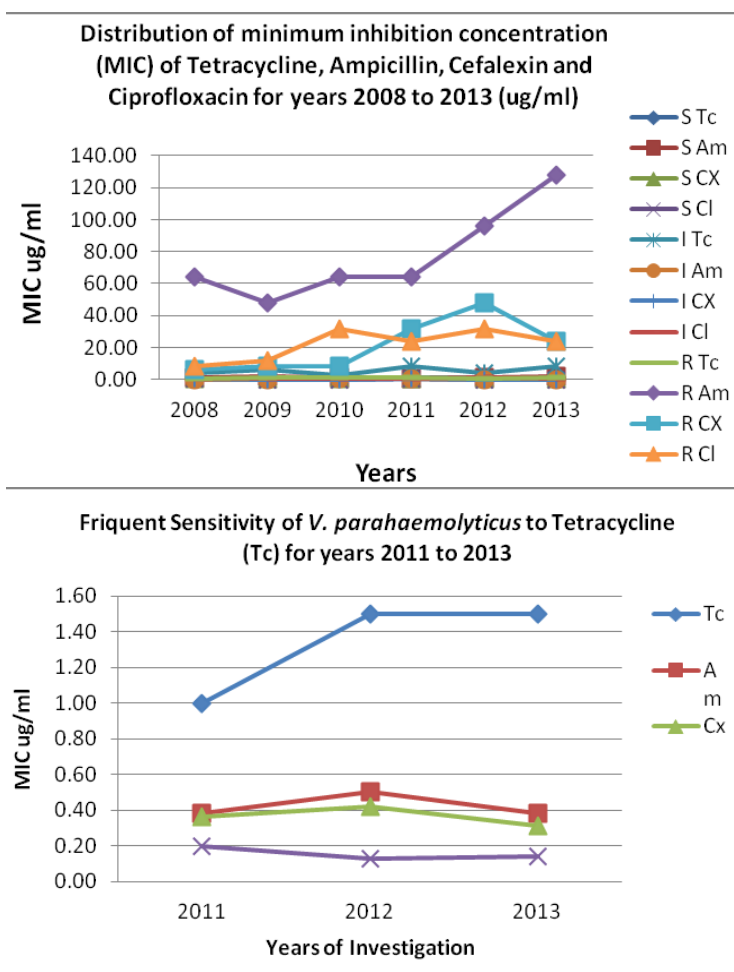

Friquent Resistance of $V$. parahaemolyticus to Ampecilin (Am) for years 2011 to 2013

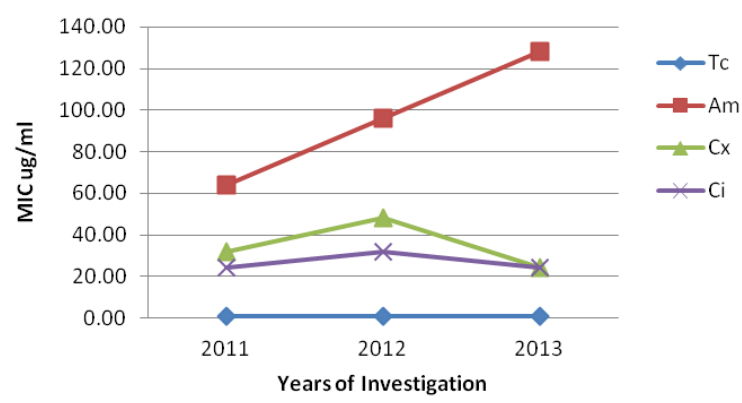

Figure 2: Distribution of Minimum Inhibition Concentration (MIC) of Tetracycline, Ampicillin, Cefalexin and Ciprofloxacin in year 2011 to 2013.

Figure 1 shows the distribution of antibiotic resistant profiles of $V$. parahaemolyticus for the four tested antibiotics from 2004 then from 2011 to 2013. The distribution showed a clear development of ampicillin resistance from maximum MIC of $64 \mu \mathrm{g} / \mathrm{ml}$ in 2011 to maximum MIC of $128 \mu \mathrm{g} / \mathrm{ml}$ in 2013 (Figure 2). The magnitude of resistance development is rapid with about 3 fold increase in the mean MIC over more than four years and continues tracing since 2004 and from this study from2011 to 2013 (Figure 3). The current study demonstrates a high risk of pathogenic $V$. parahaemolyticus in the shrimp and cockles marketed in Selangor, Malaysia.

The antibiogram obtained in current study clearly indicates that the first-line drug-tetracycline still remained highly effective against $V$. parahaemolyticus. The results showed slight decrease in the MIC of tetracycline from 2011 to 2013 suggesting the outcome of the ban of tetracycline used as a growth promoting in animal feed. Excess use of antibiotics encourages the development of antibiotic resistance and that of reduction may consequence the decrease in antibiotic resistance $[37,38]$.
Frequent Resistance of V. parahaemolyticus to Am for years 2011 to 2013

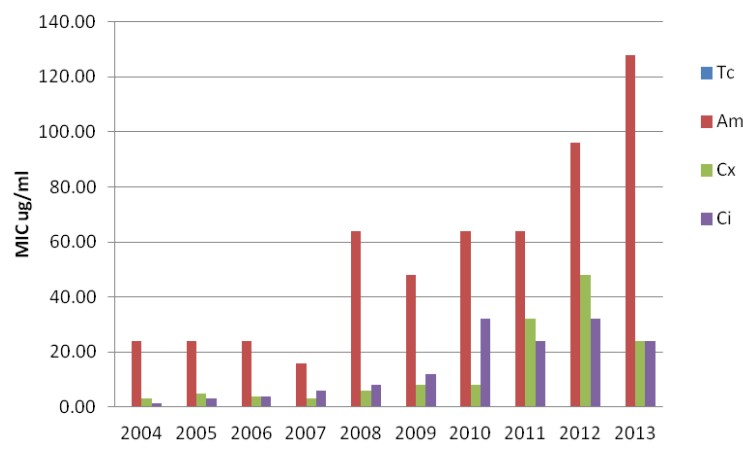

Years of the invistigation

Frequent Resistance of $V$. parahaemolyticus to Am for years 2011 to 2013

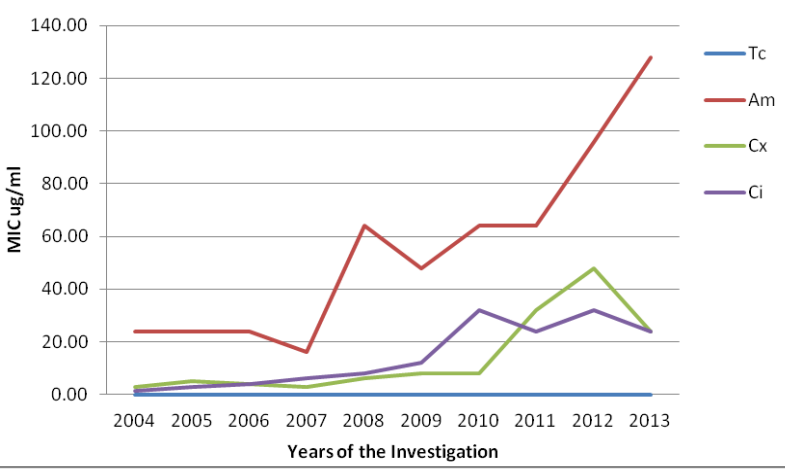

Frequent Resistance of $V$. parahaemolyticus to Am for years 2004 to 2013

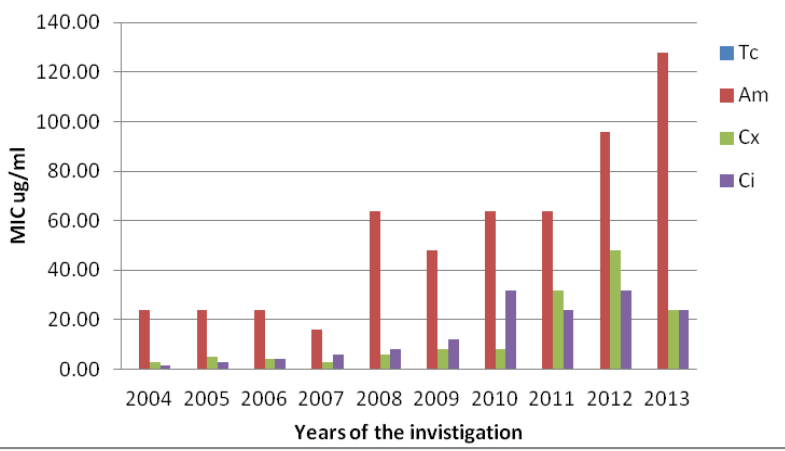

requent Resistance of V. parahaemolyticus to Am for years 2004 to 2013

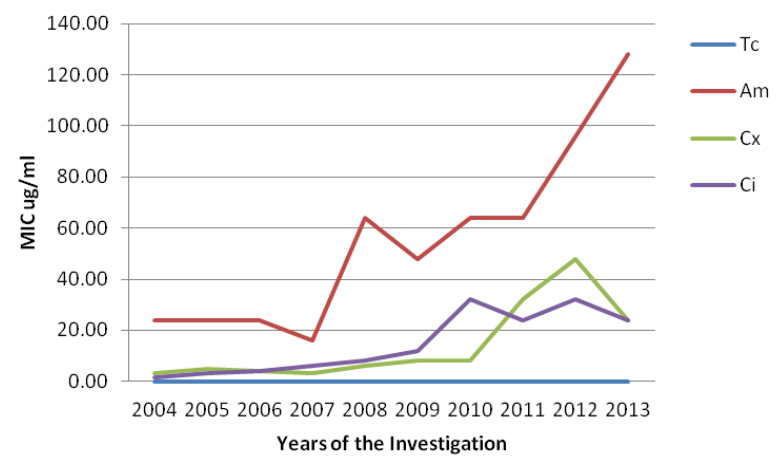

Figure 3: The increase of Ampicilin resistance in V. parahaemolyticus from 2004 and for this study from 2011 to 2013 . 
Citation: Al-Othrubi SMY, Kqueen CY, HMirhosseini CY, Hadi YA, Radu S (2014) Antibiotic Resistance of Vibrio parahaemolyticus Isolated from Cockles and Shrimp Sea Food Marketed in Selangor, Malaysia. Clin Microbial 3: 148. doi:10.4172/2327-5073.1000148

\section{Discussion}

This study is a preliminary examination of the antimicrobial susceptibilities of $V$. parahaemolyticus for retail seafood (shrimp and cockle) marketed in Selangor, Malaysia. Aquatic bacteria, including vibrios, live in the coastal and estuarine waters, an open area particularly subject to environmental contaminations by agricultural runoff or wastewater treatment plants [39], which may contain various levels of antimicrobials and heavy metals and act as selective pressure for antimicrobial-resistant aquatic bacteria $[26,34,40]$. These findings indicated that the V.parahaemolyticus strains isolated from local shrimp and cockles collected from several markets remained susceptible to the majority of antimicrobials tested; however, the observed high percentage of $V$. parahaemolyticus isolates with reduced susceptibilities to ampicillin suggests that ampicillin has a potentially low efficiency in empirical treatment of $V$. parahaemolyticus infections. Therefore, continued monitoring of both the prevalence and the antimicrobial susceptibility profile of $V$. parahaemolyticus is important to better ensure seafood safety.

From these results, the rapid method used in this study using CHROMagar Vibrio (Figure 4) compared by conventional TCBS agar medium (Figure 5) was the best to give pure colonies of $V$. parahaemolyticus within 12 to 24 hours that decrease the time wasting, cost, and efforts.

Our findings are in agreement with Wong [4] in which some environmental isolates were found to possess trh genes only but very low $t d h$ positive isolates. None of the isolates collected in this study possess both $t d h$ and trh genes suggests $t d h$ gene is mainly contained within clinical strains of $V$. parahaemolyticus. In the previous studies, the prevalence of pathogenic $V$. parahaemolyticus in the marine environment and retail seafood is relatively low. Nonetheless, there is still a potential risk of $V$. parahaemolyticus outbreak or infection through consumption of the contaminated seafood.

The $V$. parahaemolyticus food poisoning incidence in Malaysia is considerably high. However, the occurrence of pathogenic strains in

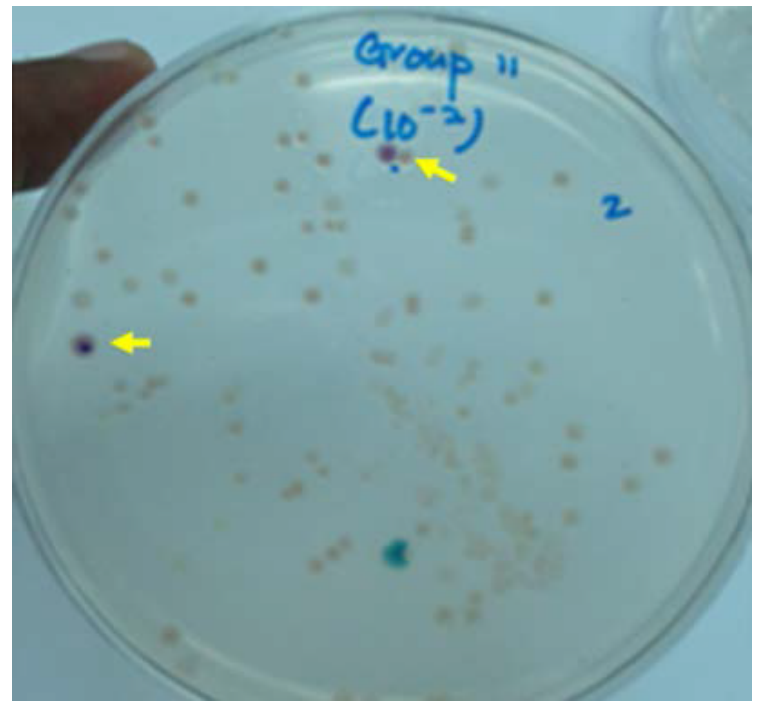

Figure 4: Colonies plated out from high selected CHROMagar Vibrio plate incubated at $37^{\circ} \mathrm{C}$ for $18-24 \mathrm{~h}$, direct and spread plating from cockles. Mauve colonies on CHROMagar Vibrio (indicated by arrow) were identified as Vibrio parahaemolyticus.

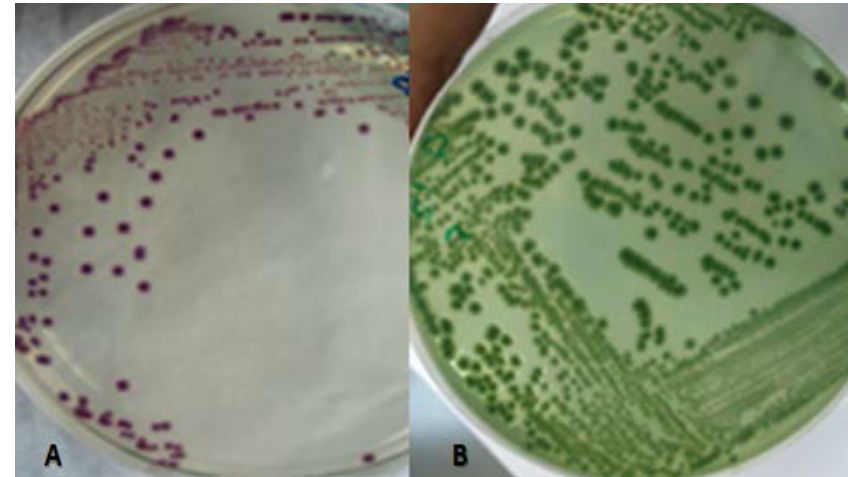

Figure 5: Pure colonies plated on CHROMagar Vibrio plates and TCBS agars Plates incubated at $37^{\circ} \mathrm{C}$ for $24 \mathrm{~h}$. Mauve colour colonies on CHROMagar Vibrio (A) and green colonies on TCBS agar (B) were identified as Vibrio parahaemolyticus.

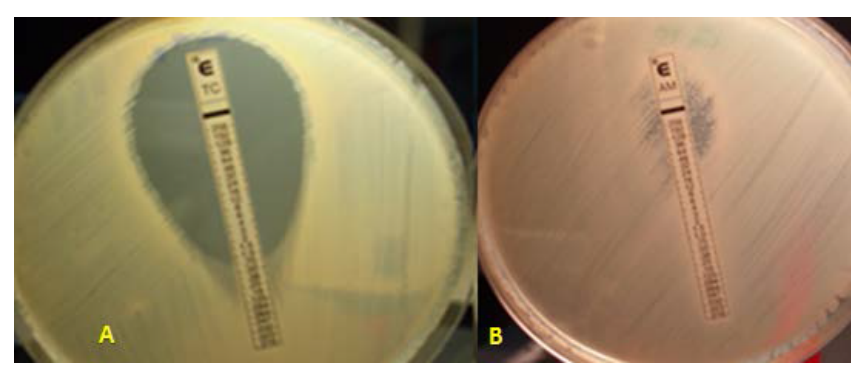

Figure 6: (A and B, the E-test plates for Tetracycline and Ampicilline antibiotics) Representative photo plates for antibiotic susceptibility E-test assay of $V$. parahaemolyticus performed on Muller Hinton agar medium showed the zoon of inhibition and MIC of the growth.

shrimp and cockles, and antibiotic resistance of $V$. parahaemolyticus strains is not well documented and studied. This study aims to provide an insight into the prevalence of $V$. parahaemolyticus strains (TDH and/or $T R H$ ) in the marine environment and retail seafood and the antibiotic resistance profile of $V$. parahaemolyticus isolated from 2004 and recently for this study from 2011 to 2013.

The antibiogram obtained in current study clearly indicates that the first-line drug-tetracycline still remained highly effective against $V$. parahaemolyticus. The results showed slight decrease in the MIC of tetracycline from 2011 to 2013 suggesting the outcome of the ban of tetracycline used as a growth promoting in animal feed. Excess use of antibiotics encourages the development of antibiotic resistance [37] and that of reduction may consequence the decrease in antibiotic resistance (Figure 6).

However, the findings in this study showed an increase in ampicillin resistance since 2004 as in our pervious study [41] and until this study from 2011 to 2013 and others [36,38]. Although ampicillin is not used empirically to treat $V$. parahaemolyticus infection in the hospital, the increase resistance rate has created a great concern. Ampicillin resistance in V. parahaemolyticus is not a new phenomenon. A 1978 study in the United States reported that over $90 \%$ of $V$. parahaemolyticus isolates were resistant to ampicillin and exhibited -lactamase activity $[42,43]$. This finding was also in agreement with a number of literatures from all around the world and Malaysia [23,44-46]. 
Citation: Al-Othrubi SMY, Kqueen CY, HMirhosseini CY, Hadi YA, Radu S (2014) Antibiotic Resistance of Vibrio parahaemolyticus Isolated from Cockles and Shrimp Sea Food Marketed in Selangor, Malaysia. Clin Microbial 3: 148. doi:10.4172/2327-5073.1000148

\section{Conclusion}

The occurrence of pathogenic $V$. parahaemolyticus in seafood and their drug resistance pattern in this study demands immediate need for paying attention. A judicious exploitation of antibiotics both for aquaculture farming and for treatment diseases should be followed to combat this drug resistance in pathogenic gram negative bacteria.

\section{Acknowledgments}

I acknowledge all my colleagues at the Center of Excellence for Food Safety Research, Faculty of Food Science and Technology, microbiology laboratory for their assistance and kind help during my lab work and data analysis specially my colleagues; Alex, Ubong, Goh Sur, Lye Ying Ling, and all the others of my colleagues. Special thanks go to Prof. Dr. Son Radue for his kind guidance for this work, and same thanks go to Assoc. Prof. Dr. Cheah Yoke Kqueen, faculty of Medicine for his kind help since early of this study. And finally; special thanks go to Assoc. Prof. Dr. Hamed Syed for his kind help to check our statistic analysis.

\section{References}

1. Shigeaki M, Natsumi O, Toshio K, Takeshi H, Tetsuya I (2012) A Cytotoxic Type III Secretion Effector of Vibrio parahaemolyticus Targets Vacuolar H+ -ATPase Subunit c and Ruptures Host Cell Lysosomes. PLoS Pathogens.

2. DePaola A, Ulaszek J, Kaysner CA, Tenge BJ, Nordstrom JL, et al. (2003) Molecular, serological, and virulence characteristics of Vibrio parahaemolyticus isolated from environmental, food, and clinical sources in North America and Asia. Appl. Environ. Microbiol 69: 3999-4005.

3. Joseph SW, Colwell RR, Kaper JB (1982) Vibrio parahaemolyticus and related halophilic Vibrios. Critic Rev Microbiol 10: 77-124.

4. Wong HC, Liu SH, Wang TK, Lee CL, Chiou CS, et al. (2000) Characteristics of Vibrio parahaemolyticus O3:K6 from Asia. Appl Environ Microbiol 66: 3981 3986.

5. Yeung PS, Boor KJ (2004) Epidemiology, pathogenesis, and prevention of foodborne Vibrio parahaemolyticus infections. Foodborne Pathog Dis 1: 74-88.

6. Kam MK, Luey CK, Parsons MB, Nair GB, Alam M, et al. (2008) Evaluation and Validation of a PulseNet Standardized Pulsed-Field Gel Electrophoresis Protocol for Subtyping Vibrio parahaemolyticus: an International Multicenter Collaborative Study. J Clin Microbiol 46: 2766-2773.

7. Maluping RP, Lavilla PCR, DePaola A, Janda JM, Krovacek K, et al. (2005) Antimicrobial susceptibility of Aeromonas spp., Vibrio spp. and Plesiomonas shigelloides isolated in the Philippines and Thailand. Int J Antimicrob Agents 25: $348-350$

8. Martinez UJ, Huapaya B, Gavilan RG, Blanco AV; Ansede BJ, et al. (2008) Emergence of Asiatic Vibrio Diseases in South America in Phase With El Nino. Epidemiology 19: 829-837.

9. Francesco R, Joseph PYK, Carla F, Alessia F, Gianfranco D, et al. (2000) Enterotoxicity and Cytotoxicity of Vibrio parahaemolyticus Thermostable Direct Hemolysin in In Vitro Systems. Infect Immun 68: 3180-3185.

10. Itaru Y, Kumiko N, Tsutomu Y, Shuji K, Kouta M, et al. (2010) Structure and Functional Characterization of Vibrio parahaemolyticus Thermostable Direct Hemolysin. J Biol Chem 285: 16267-16274.

11. María ECG, Carlos Vázquez-S, Quiñones-Ramírez El (2004) Serologic and Molecular Characterization of Vibrio parahaemolyticus Strains Isolated from Seawater and Fish Products of the Gulf of Mexico. Applied and Environmental Microbiology 70: 6401-6406.

12. Honda S, Goto I, Minematsu N, Ikeda N, Asano N, et al. (1987) Gastroenteritis due to Kanagawa negative Vibrio parahaemolyticus. Lancet 1: 331-332.

13. J, Ishibashi M, Abbott SL, Janda JM, Nishibuchi M (1997) Analysis of the thermostable direct hemolysin $(t d h)$ gene and the tdhrelated hemolysin (trh) genes in urease-positive strains of Vibrio parahaemolyticus isolated on the West Coast of the United States. J Clin Microbiol 35: 1965-1971.

14. Su YC, Liu C (2007) Vibrio parahaemolyticus: A concern of seafood safety Food Microbiology 24: 549-558

15. Caburlotto G, Ghidini V, Gennari M, Tafi MC, Lleo MM (2008) Isolation of a Vibrio parahaemolyticus pandemic strain from a marine water sample obtained in the northern Adriatic. Eurosurveillance.
16. Nordstrom JL, Vickery MC, Blackstone GM, Murray SL, DePaola A (2007) Development of a multiplex real-time PCR assay with an internal amplification control for the detection of total and pathogenic Vibrio parahaemolyticus bacteria in oysters. Appl Environ Microbiol 73: 5840-5847.

17. Oliver JD (2006) Vibrio vulnificus. The biology of vibrios. ASM Press, Washington, DC

18. Cabello FC (2006) Heavy use of prophylactic antibiotics in aquaculture: a growing problem for human and animal health and for the environment. Environ Microbiol 8: 1137-1144.

19. Mazel D, Davies J (1999) Antibiotic resistance in microbes. Cell Mol Life Sci 56: 742-754.

20. Morris JG, Tenney J (1985) Antibiotic therapy for Vibrio vulnificus infection. JAMA 253: 1121-1122.

21. Tang HJ, Chang MC, Ko WC, Huang KY, Lee CL, et al. (2002) In vitro and in vivo activities of newer fluoroquinolones against Vibrio vulnificus. Antimicrob. Agents Chemother 46: 3580-3584.

22. Abdul-Rahim M, Jamal KH, Son R (2007) Joint Food Safety and Quality Division, Ministry of Health Malaysia/ National Food Safety Research Center Faculty of Food Science and Technology, University Putra Malaysia Expert Consultation on Risk Assessment of Vibrio parahaemolyticus in Black Tiger Prown (Penaeus monodon).

23. Marlina, Son R, Cheah YK, Suhaimi N, Zunita Z, et al. (2007) Occurrence of $t d h$ and trh genes in Vibrio parahaemolyticus isolated from raw and processed bivalves (Corbicula molktiana), in West Sumatera, Indonesia. South East Asian Journal of Tropical Medicine and Public Health 38: 349-355.

24. Nasreldin E, Son R, Chien HC, Mitsuaki N (2004) Prevalence of Potentially Pathogenic Vibrio Species in the Seafood Marketed in Malaysia. Journal of Food Protection 67: 1469-1475.

25. Nishibuchi M, Fasano A, Russell RG, Kaper JB (1992) Enterotoxigenicity of Vibrio parahaemolyticus with and without genes encoding thermostable direct hemolysin. Infect Immun 60: 3539-3545.

26. Stepanauskas R, Glenn TC, Jagoe CH, Tuckfield RC, Lindell AH, et al. (2006) Coselection for microbial resistance to metals and antibiotics in freshwater microcosms. Environ Microbiol 8: 1510-1514.

27. Miyamoto Y, Kato T, Obara Y, Akiyama S, Takizawa K, et al. (1969) In vitro hemolytic characteristic of Vibrio parahaemolyticus: its close correlation with human pathogenicity. J Bacteriol 100: 1147-1149

28. Nishibuchi M, Kaper JB (1985) Nucleotide sequence of the thermostable direct hemolysin gene of Vibrio parahaemolyticus. J Bacteriol 162: 558-564.

29. Cook DW, O'Leary P, Hunsucker JC, Sloan EM, Bowers JC, et al. (2002) Vibrio vulnificus and Vibrio parahaemolyticus in U.S. retail shell oysters: a national survey June 1998 to July 1999. Journal of Food Protection 65: 79-87.

30. DePaola A, Kaysner CA, Bowers J, Cook DW (2000) Environmental investigations of Vibrio parahaemolyticus in oysters after outbreaks in Washington, Texas, and New York (1997 and 1998). Appl Environ Microbio 66: 4649-4654

31. Thompson CA, Vanderzant C (1976) Relationship of Vibrio parahaemolyticus in oysters, water and sediment, and bacteriological and environmental indices. Journal of Food Science 41: 118-122.

32. Kaysner CA, Abeyta C, Stott JRF, Krane MH, Wekell MM (1996) Enumeration of Vibrio species, including $V$. cholerae from samples of an oyster growing area, Grays Harbor, Washington. J Food Prot 53: 302-304.

33. Honda T, Ni Y, Miwatani T (1988) Purification and characterization of a hemolysin produced by a clinical isolate of Kanagawa phenomenon-negative Vibrio parahaemolyticus and related to the thermostable direct hemolysin. Infect Immun 56: 961-965.

34. Han F, Walker RD, Janes ME, Prinyawiwatkul W, Ge1 B (2007) Antimicrobia Susceptibilities of Vibrio parahaemolyticus and Vibrio vulnificus Isolates from Louisiana Gulf and Retail Raw Oysters. Appl Env Microbiol 73: 7096-7098.

35. (CDC) Centers for Disease Control and Prevention (2005) posting date. Vibrio parahaemolyticus. Disease listing. Centers for Disease Control and Prevention, Atlanta, GA

36. Hussein S (2010) Determination of Minimum Inhibitory Concentrations of Antibiotics by E-test. American Society for Microbiology (ASM MicrobeLibrary).

37. Marcus HYW, Ming L, Hoi YW, Sheng C (2012) Characterization of Extended- 
Citation: Al-Othrubi SMY, Kqueen CY, HMirhosseini CY, Hadi YA, Radu S (2014) Antibiotic Resistance of Vibrio parahaemolyticus Isolated from Cockles and Shrimp Sea Food Marketed in Selangor, Malaysia. Clin Microbial 3: 148. doi:10.4172/2327-5073.1000148

Page 7 of 7

Spectrum- $\beta$-Lactamase-Producing Vibrio parahaemolyticus. Antimicrob. Agents Chemother 56: 4026-4028.

38. Molla B, Mesfin A, Alemayehu D (2003) Multiple antimicrobial-resistan Salmonella serotypes isolated from chicken carcasses and giblets in Debre Zeit and Addis ababa, Ethiopia. Ethiopian J Health 17: 131-149.

39. Kristi SS, Rachel ERG, Xin H, John MJ, Byron CC, et al. (2014) Antimicrobia Susceptibility of Vibrio vulnificus and Vibrio parahaemolyticus Recovered from Recreational and Commercial Areas of Chesapeake Bay and Maryland Coastal Bays. PLOS ONE 9: e89616.

40. Gordon L, Giraud E, Ganiere JP, Armand F, Bouju-Albert A, et al. (2007) Antimicrobial resistance survey in a river receiving effluents from freshwater fish farms. J Appl Microbiol 102: 1167-1176.

41. Saleh Al-Othrubi M, Alfizah H, Son R, Humin N, Rahaman J (2011) Rapid detection and E-test antimicrobial susceptibility testing of Vibrio parahaemolyticus isolated from seafood and environmental sources in Malaysia. Saudi Med J 32: 400-406.
42. Van Leeuwen WJ, Van Embden J, Guinee P (1979) Decrease of Drug resistance in Salmonella in the Netharlands. Antimicrob Agents Chemother 16: 237-239.

43. Joseph SW, DeBell RM, Brown WP (1978) In vitro response to chloramphenicol, tetracycline, ampicillin, gentamicin, and beta-lactamase production by halophilic vibrios from human and environmental sources. Antimicrob Agents Chemother 13: $244-248$.

44. Nettip N, Suthienkul O, Eampokalap B (1992) Presented at the XIIIth International Congress for tropical Medicine and Malaria. Ambassador Hotel, Jomtien, Pattaya, Thailand.

45. Orlando (2003) Discussion paper on risk management strategies for Vibrio Spp In Seafood. Joint Fao/Who Food Standards Programme Codex Committee on Food Hygiene, USA.

46. Pumiprapat J, Suthienkul O, Siripanichagon K (1993) Presented at the World Congress on Tourist Medicine and Health, The Mandarin Hotel, Singapore. 